\title{
Modulation structures in the dynamic spectra of Jovian radio emission obtained with high time-frequency resolution
}

\author{
G. V. Litvinenko ${ }^{1}$, A. Lecacheux ${ }^{2}$, H. O. Rucker ${ }^{3}$, A. A. Konovalenko ${ }^{1}$, B. P. Ryabov ${ }^{1}$, U. Taubenschuss ${ }^{3}$, \\ V. V. Vinogradov ${ }^{1}$, and V. E. Shaposhnikov ${ }^{4}$
}

\author{
1 Institute of Radio Astronomy, Kharkov, Ukraine \\ e-mail: gallitv@rian.kharkov.ua \\ 2 CNRS \& Observatoire de Paris, France \\ 3 Space Research Institute, Graz, Austria \\ 4 Institute of Applied Physics, Nizhny Novgorod, Russia
}

Received 28 February 2008 / Accepted 16 September 2008

\section{ABSTRACT}

\begin{abstract}
Aims. The wide-band dynamic spectra of Jovian decameter emission obtained over the last decade with high-frequency and high time resolution equipment on the largest decameter band antenna array, the Ukrainian T-shape Radio telescope (UTR-2), are presented. Methods. We analyzed the data obtained with the Digital SpectroPolarimiter (DSP) and WaveForm Reciever (WFR) installed at UTR-2. The combination of the large antenna and high performance equipment gives the best sensitivity and widest band of analysis, dynamic range, time and frequency resolutions. The wavelet transform method and the Fourier technique was used for further data processing.

Results. The main characteristics of already known and newly detected modulation events were investigated and specified. The new receiving-recording facilities, methodology and program of observations are described in detail.
\end{abstract}

Key words. planets and satellites: individual: Jupiter

\section{Introduction}

Slightly more than 50 years ago Burke \& Franklin (1955) discovered non-thermal sporadic radio emission from Jupiter at 22.2 MHz. The Jovian emission covers the decameter frequency range from several $\mathrm{MHz}$ up to about $40 \mathrm{MHz}$. Currently, despite continuing interest in the investigation of this phenomenon with ground-based methods (see, for instance, Nigl et al. 2007; Hess et al. 2007b; Taubenschuss et al. 2006) as well as by space missions (see, for instance Hospodarsky et al. 2004; Spilker et al. 2007; Mutel et al. 2007) there is no complete theory able to explain the origin of the radiation and the particularities appearing in the dynamic spectra of DAM (decameter) emission (Hess et al. 2007a; Zarka et al. 2007; Shaposhnikov et al. 2008). Jupiter is among the five presently known outer planets that have radio sources associated with their auroral zones. A review of both observational and theoretical aspects of the auroral radio emission from the outer planets is given in the works of Zarka (Zarka 1998, 2004). Jupiter is apparently the only radio planet from which non-thermal magnetospheric radio emission is accessible from the Earth's surface. According to the modern classification, the Jovian DAM emission consists of two types of wide band radio storms, called L- and S-bursts, which are different in the radio burst spectral envelopes and time scales (L - Long - the time scale $1 \div 100 \mathrm{~s} ; \mathrm{S}$ - Short - the time scale $1 \div 100 \mathrm{~ms}$ ). It is supposed that the time scales of the L-radiation are due to radiation scattering on the interplanetary plasma and the Earth's ionosphere irregularities. The S-burst time scales mainly can be attributed to the properties of the radiation source. Their occurrence and intensity are strongly dependent on the orbital position of the Jovian satellite Io. Monitoring of the Io-dependent DAM emission revealed the characteristic Jupiter emission zones known as Io-A, Io-B, Io-C, and Io-D (Flagg \& Desch 1979; Goertz 1976; Genova \& Leblanc 1981; Litvinenko et al. 2000; Ryabov 1992). One of the most interesting particularities of S-bursts is their rapid drift in the frequencytime plane, which has no reliable explanation (see Ryabov \& Gerasimova 1990, and reference therein). Electromagnetic radiation represents a fast extraordinary wave. It is suggested that the emission pattern is a cone with radiation in the cone mantle and the apex angle that is directed towards the planet. Since the brightness temperature of the radio bursts is substantially higher than the temperature of the Jovian magnetosphere and ionosphere, it is supposed that the generation mechanism is coherent (Hess et al. 2007b; Ryabov \& Gerasimova 1990; Herbert et al. 1967; Melrose 1986; Calvert \& Ellis 1988; Willes 2002; Zaitsev et al. 1986).

The Jovian S-bursts represent a powerful radio emission in the Solar system and study of thir properties may guide the search for Jupiter-like decameter radio bursts from exoplanets (Zarka 2004, 2006).

In spite of the more than 50 years of extensive exploration of the Jovian DAM radiation, the different kinds of modulation effects on the decameter dynamic spectra have not be much studied. Several authors have investigated the modulation events, and some of their results can be found in Gordon \& Warwick (1967); Riihimaa (1968, 1970, 1978, 1979, 1988, 1991); Riihimaa et al. (1970); Genova et al. (1981); Zheleznyakov \& Shaposhnikov (1979); Meyer-Vernet et al. (1981); Warwick \& Dulk (1964); Lecacheux (1976); Ladreiter et al. (1995); Imai et al. (1997); Arkhypov \& Rucker (2007). For instance, Faraday rotation of the polarization plane in the Jupiter decameter signal was 
considered in detail by Warwick \& Dulk (1964); Lecacheux (1976); Ladreiter et al. (1995); Shaposhnikov et al. (1999). Riihimaa (1968, 1970, 1979) described the effect of the "modulation lanes", groups of lanes drifting in the time-frequency plane. He found that in the spectra these lanes have positive or negative drift, or, in some cases, both kinds of drift superposed. Firstly Riihimaa (1968, 1970) and then Imai et al. (1997) proposed an "interference screen" model to explain the origin of modulation lanes. Genova et al. (1981) found a new class of modulations, called "high frequency lanes", which appear in the high frequency part of the dynamic spectrum. Moreover, these authors, based on the analysis of much experimental data, suggested that modulation events are either produced by the Earth's ionosphere or by the oscillations in interplanetary medium. Riihimaa (1991) examined 20 high-resolution spectra of S-bursts observed at 20-30 MHz recorded in the Io-B region. He considered the temporal changes of wide-range S-bursts storms as well as the evolution of the spectral fine structure over the storm. It was shown, for instance, that "some kinds of storms start as two narrow-range $(200-500 \mathrm{kHz}) \mathrm{S}$-trains evolving into three trains, which then combine in a stepwise manner with widerange S-bursts (actually the main S-bursts of tilted- $\mathrm{V}$ events), and finally merge together to form a wide-range quasi-periodic storm." In the work of Ryabov (2001) the evolution of the Sburst fine structure (Io-B and Io-C sources) was studied against a background of multi-scale spectra of Io-dependent radio-burst emission. The author reported a repetition of the self-similar burst structures at different scales on the frequency-time plane. Carr \& Reyes (1999) and Litvinenko et al. (2004), using different specific procedures of time resolution improvement (to $1 \mu \mathrm{s}$ ), showed that the simple Jovian S-bursts have an internal microstructure that also shows modulation of the DAM emission. Later on Ryabov et al. (2007) proposed a "narrow-band filter applied to random noise" to model the microsecond structure features.

Attention in the present work is centered on the modulation effects (periodic and quasi-periodic phenomena) observed in the dynamic spectra of the Jovian decameter emission. Arcs, rotational and orbital modulation do not need high sensitivity and high frequency time resolution measurements and are not discussed here. The detailed consideration of the physical mechanisms responsible for either modulation event also is outside the scope of this work.

The lack of investigations examining the modulation events on different time scales can be explained by the following: first, the experimental equipment used had low sensitivity, narrow dynamic range and low resolution, second, the mathematical methods for data processing were not fully adapted to defining fine-scale sporadic, non-stationary structures. In the last decade new high sensitivity recording facilities, such as the digital spectro-polarimiter (DSP) (Kleewein et al. 1997) and waveform receiver (WFR) (Leitner \& Rucker 2001) have been developed. Here we analyze the data obtained with the DSP and WFR installed at the largest existing decameter band antenna array UTR-2 (Ukrainian T-shape Radio telescope, second modification, Kharkov, Ukraine, see for details Konovalenko et al. (2001)). The current combination (antenna + spectral equipment) gives the best sensitivity, band of analysis, dynamic range, and time and frequency resolution. This allows us to reveal the hierarchy of modulation, "size scaled" in the time and frequency domains. For data processing the wavelet transform method as well as the Fourier technique we was used. The classical Fourier technique is a very useful signal processing method, but its main drawback is the impossibility of simultaneously obtaining the localization of a signal in the time and frequency domains because of the "uncertainty relation". In contrast, the wavelet transform allows the dividing of data, functions or operators into different frequency components, and then considers each component with a resolution matched to its scale (Daubechies 1988). The wavelet transform is able to "zoom in" on very short-lived high frequency phenomena, such as local periodicity or nonstationarity in the signals. So, the use of wavelet analysis allows us to investigate very fine structures of the Jovian DAM signal.

\section{Equipment and methods of observation}

For many years of Jovian decameter emission studies, much fundamental information has been obtained with comparatively small antennae with an effective area of the order of only several tens of square meters (small-sized array, log-periodic array and so on.). The Riihimaa works (Riihimaa 1968, 1970, 1978, 1979, $1988,1991)$ are an example of such fruitful investigations. He effectively studied the Jovian signals with a 4-element antenna with a sensitivity of only $10^{5} \mathrm{Jy}$. This is not suprising as many characteristic features of Jupiter radiation have a flux density of $10^{7}-10^{8} \mathrm{Jy}$.

At the same time, as it was mentioned in the Introduction, a complete theory of the Jovian decameter emission does not exist, and a more detailed interpretation of the obtained results as well as further development of the theory is needed. However, more investigations using the newer, more sensitive instrumens are also very important. Discovering more weak events and the less contrasting fine time-frequency structures may provide new information about the nature and physical mechanism of the Jovian radio emission in general.

\subsection{Radio telescopes}

Currently the UTR-2 radio telescope (Braude et al. 1978) is the world's largest radio astronomical instrument in the decameter wave range. The instrument has a large linear size and effective area, a broad frequency band, high directivity, low side lobes, a multi-beam regime, electronic beam steering, a broad dynamic range, a linear tract, interference immunity, a flexible configuration and a sophisticated control system.

In spite of the smaller size relative to UTR-2, the NDA (Nancay Decameter Array, France), due to its high broad-band frequency range, electronic beam steering, and the possibility of polarization measurements, is also well adapted to a Jupiter decameter emission study (Boischot et al. 1981).

\subsection{Digital spectral processors}

Large radio astronomical antennae require adequate receivingrecording equipment. In the last 5-10 years the progress in electronic, digital and computer techniques opened the possibility of the creation of a new generation of effective digital recording systems. To obtain the most informative observations of Jovian DAM emission the required parameters are: 1) the frequency band of the simultaneous analysis must be equivalent to the receiving emission band ( $\sim 10-40 \mathrm{MHz})$, i.e. $>10 \mathrm{MHz} ; 2)$ time and frequency resolutions need to be $\sim 1 \mathrm{~ms}$ (or less) and of the order of $1-100 \mathrm{kHz}$ (number of equivalent frequency channels >1000); 3) dynamic range (maximum to minimum signal intensity) should be $10^{5}$. The modern analog to digital converter (ADC), spectral processors with $12-16$ bits and a sampling frequency of a few tens of megagertz, a PC with a sampling frequency of more than $1 \mathrm{GHz}$ and so on, allow us to obtain 
the above-mentioned requirements for a high sensitivity and high spectral resolution observation of the Jovian sporadic decameter signals. A similar approach was used for the development of digital spectral processors (DSP) (Kleewein et al. 1997). Such devices are used on the existing decameter telescopes Nancay/France and Kharkov/Ukraine.

The analog part of the DSP provides an amplification, transformation and filtration of the signal which comes from the telescope, and then automatically creates in real time a Fourier spectrum that is produced by integration and averaging processes. The DSP can operate down to the time resolution of one millisecond with 1024 frequency channels over an instantaneous bandwidth of $12.5 \mathrm{MHz}$ and is able to measure a full wave polarization. The frequency resolution is fixed at $\delta f=12.5 \mathrm{kHz}$. The analog-digital converter (ADC) has a resolution of $b=12$ bits, which gives a dynamic range $D_{\mathrm{dB}} \approx 6 \times b \approx 72 \mathrm{~dB}\left(\sim 10^{7}\right)$, and works with the sampling frequency $F_{\mathrm{s}}=2 \times B=25 \mathrm{MHz}$ (where $B$ is the frequency band of $12.5 \mathrm{MHz}$ within the input frequency range of $f_{0}=5 \ldots 50 \mathrm{MHz}$ ). To obtain high-quality spectra for events of any intensity (from the maximum possible $10^{8} \mathrm{Jy}$ to the minimum observable $10^{2} \mathrm{Jy}$ ) it is necessary to have a dynamic range of approximately $70 \mathrm{~dB}$.

Although the above described equipment is highly effective, there are problems which need another approach. Among them is the investigation of specific fine properties of Jovian DAM emission. The drawbacks of DSP in this case are the limitation of time resolution (1 ms) and loss of signal phase information. This loss of information makes the Fourier transform an operation which cannot be inverted. Thus, it is necessary to record the whole time series. This could be done in an analog or a digital way. A digital approach is more useful because of a larger bandwidth and dynamic range. However, the amount of data produced is enormous and thus loss-free data compression needs to be applied in this approach.

\subsection{Waveform receiver}

The current level of technical development has allowed a solution of this compression problem. Using the fast-acting ADC and large memory arrays it is possible to record an initial signal of a certain duration. Such an instrument, called a waveform receiver (WFR), was based an analog part of the DSP, the ADC with a sampling frequency of $50 \mathrm{MHz}$ and 100 megabyte per second data generated which is managed by a PCI - bus system (Peripheral Component Interconnect) (Leitner \& Rucker 2001). The WFR reaches a theoretical maximum possible timefrequency resolution. The main concept of the waveform receiver is to store the signal's original waveform. The digitized data is transferred via the PCI-bus to the internal memory of a standard personal computer system. The amount of memory available defines the maximum length of signal. The $650 \mathrm{MB}$ of internal memory of the WFR setup allows the recording of a signal with a duration of $6.5 \mathrm{~s}$. For further data processing the wavelet transform method is used. The mathematical properties of the method give the possibility of obtaining a very high time resolution (significantly less than $1 \mathrm{~ms}$ ).

DSP and WFR were installed at the telescopes UTR-2 and NDA from 1996 to 2002 (Rucker et al. 2001) and we used for the Jovian emission observations. The experiments were carried out according to the Io-controlled S-bursts emission scheme. In some cases observations were organized simultaneously at UTR-2 and NDA with precise time synchronization $(<5 \mathrm{~ms})$. The duration of the daily observation session was several hours during the night and day. The common number of sessions was 30 . The time resolution in the joint experiments varied from 1 to $100 \mathrm{~ms}$. A total of $300 \mathrm{~GB}$ of data were obtained. Processing was performed by IDL. As a result the twodimensional array (time-frequency dependence) is expressed as a brightness dynamic spectrum which is used in subsequent investigations.

\section{Results}

The analysis of a large amount of Jovian sporadic decameter emission represented in dynamic spectra leads to the conclusion that practically all observed radiation is in some way modulated. In each specific case however the nature of the modulation can be different. A mechanism that generates Jovian bursts can itself create modulation processes and the initial emission characteristics can be changed along the propagation path from the source region to the observer (Jupiter's magnetosphere, the interplanetary medium, Earth's ionosphere). Depending on the time and frequency resolution achieved in the experiment and also on the visualization time scale, different features of the radiation spectra appear in the Jovian dynamic spectra. This is the reason for difficulties in data analysis and interpretation. In Fig. 1 the consecutive zooms of one dynamic spectrum are presented. An initial spectrum was obtained with NDA and DSP. The spectrum images changed by varying the visualization scale in the frequency domain as well as the time interval.

In the present work a set of Jovian dynamic spectra with time scales shorter than $\sim 10$ min obtained with high frequency and time resolution equipment (DSP, WFR) are considered. The major effort centers on the definition of a full set of modulation effects which appear in the spectra, of known types as well as those observed for the first time.

\subsection{Faraday fringes}

During the observation of the Jovian DAM emission with linear polarized antennae (for instance, with the UTR-2 antenna array) the system of dark and light lanes, horizontal ${ }^{1}$ over the time axis the so-called Faraday fringes (Fig. 2), is always present in the dynamic spectra. The Jovian decameter radio emission is a strong elliptical polarized emission and travels from Jupiter to the Earth through several magnetized media: Io's plasma torus, the interplanetary medium and the Earth's ionosphere (Shaposhnikov et al. 1999). The elliptically polarized emission propagating through the magnetized plasma separates into two coherent components corresponding to the normal modes (extraordinary and ordinary) when a geometric-optic approximation is valid. During this propagation, in particular through the Earth's ionosphere, the phase velocity and the ray path are different for the two modes, resulting in a rotation of the ellipse axis, the so-called Faraday rotation. Using linearly polarized antennae, the recorded Jovian dynamic spectra are modulated by fringes due to this rotation. In this case the modulation depth is dependent on the order of the radiation ellipticity, i.e. on the ratio of the main axis of the polarization ellipse. From the discussion

\footnotetext{
1 Sometimes slight frequency drift of Faraday fringes is observed which can be explained by the temporal variation of the plasma parameters (mainly by the changing of the electron density) along the DAM emission propagation in the Earth's ionosphere and Io's plasma torus (Shaposhnikov et al. 1999). For example, in Fig. 2 the frequency drift of Faraday fringes is $\sim 0.05 \mathrm{MHz} / \mathrm{min}$, which may be caused, for instance, by the temporal variation of the electron density of the Earth's ionosphere of the order of $\sim 0.4 \%$ per min.
} 

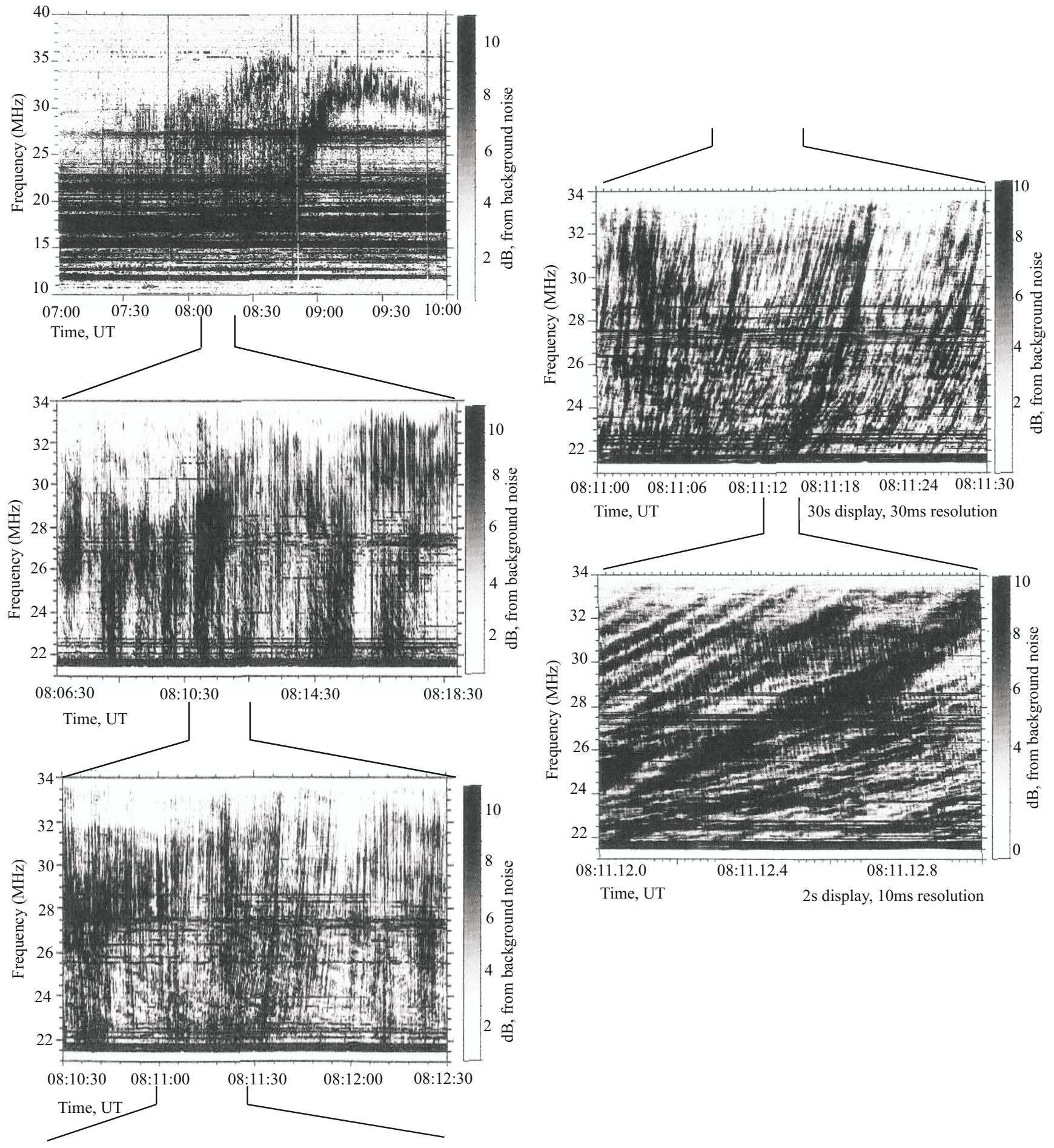

Fig. 1. Images of the Jovian DAM emission spectra on different time scales (NDA + DSP, Io-B source).

above it is clear that the Faraday fringes cannot be observed by capturing the Jovian DAM emission with a circular polarized antenna. Warwick \& Dulk (1964) were the first who proposed an explanation for these modulation lines in the Jovian DAM dynamic spectra by the effect of wave propagation. The amount of Faraday rotation is proportional to the plasma density along the emission path and depends on frequency. Therefore the observations of Faraday fringes can be used to remotely sense the electron content of the medium traversed by the wave. Moreover, this effect gives us the possibility of monitoring the latitudinal inhomogeneity of the Io plasma torus (Shaposhnikov et al. 1999). It is not yet clear what the percentage ratio is of each medium's (Io's plasma torus and the Earth's ionosphere) contribution to the full Faraday rotation. To resolve this issue it is necessary to provide simultaneous space and ground-based observation. The works of Parker et al. (1969) and Boudjada \& Lecacheux (1991) have made an attempt to use Faraday fringes for estimation of the initial orientation of the major axis of the polarization angle of the emission to the planetary magnetic field in the source region. However, the results of this investigation are contradictory. It was shown in Shaposhnikov et al. (1999) that the existence of the frequency limit above which there is no decameter radiation 


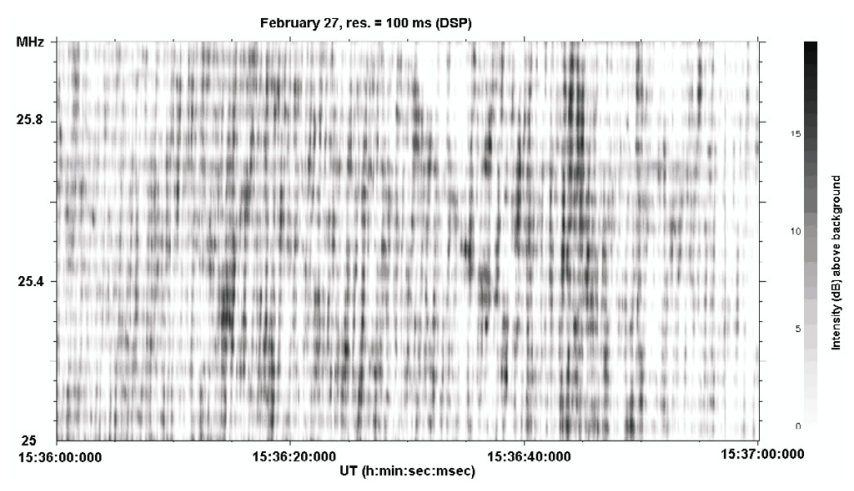

Fig. 2. Faraday fringes observed in the dynamic spectrum of the Jovian DAM emission (UTR-2 + DSP, Io-B source).

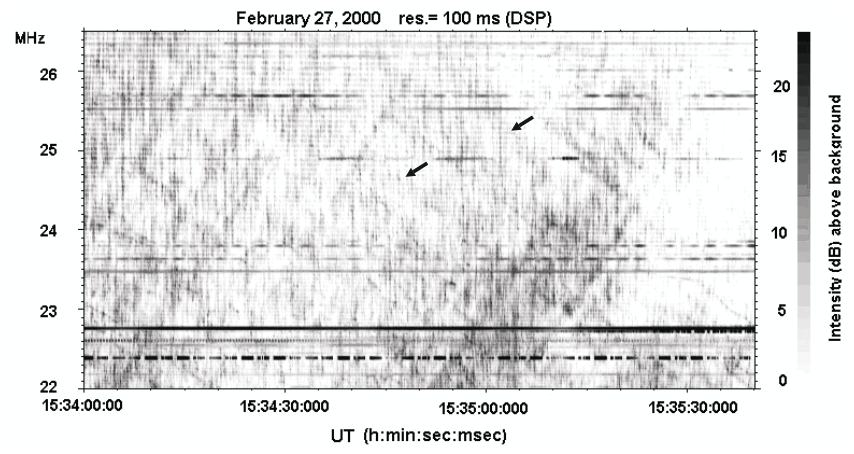

Fig. 3. Dynamic spectrum of the Jovian DAM emission with a modulation structure L3 (UTR-2 + DSP, Io-B source).

from Jupiter confines the precise calculation of the polarized ellipse orientation in the source.

\subsection{Modulation lanes}

Another kind of modulation present in the Jovian dynamic spectra are groups of lanes with high and low intensity drifting in the time - frequency plane, so-called modulation lanes (Gordon \& Warwick 1967; Riihimaa 1968, 1970, 1978, 1979, 1988, 1991; Riihimaa et al. 1970; Genova et al. 1981). According to Riihimaa, who first discovered and intensively studied this phenomenon (Riihimaa 1968, 1970, 1978, 1979, 1988; Riihimaa et al. 1970), the dynamic spectra with modulation lanes can be divided into three types, L2 with positive drifting lanes (radiation frequency rises over time), L4 with negative drifting lanes, and L3, which is a superposition of L2 and L4.

Figures 3 and 4 illustrate events L3 and L2, respectively. In Fig. 3 the following modulations are clearly visible: faraday fringes (the structure of interchangeable dark and light horizontal parallel lanes whose density is inversely proportional to the square of the frequency) and modulation lanes with different drift rates. In Fig. 3 the magnitude of the frequency drift is practically the same for lanes with positive and with negative inclines (about $0.1 \mathrm{MHz} / \mathrm{s}$ ), but the lanes show different curvatures. The frequency drift of the lanes (structure L2) in Fig. 4 is on average $0.15 \mathrm{MHz} / \mathrm{s}$. Our investigations of modulation lanes partly confirm the following statements present in the literature: 1) the classical modulation lanes generally cover the whole frequency range; 2) the usual frequency spacing between the nearest modulation lanes is approximately $100-300 \mathrm{kHz} ; 3$ ) the average frequency drift rate is $100 \mathrm{kHz} / \mathrm{s}$; 4) the modulation depth is a few $\mathrm{dB} ; 5)$ the sign of drift has a very strong dependence on the

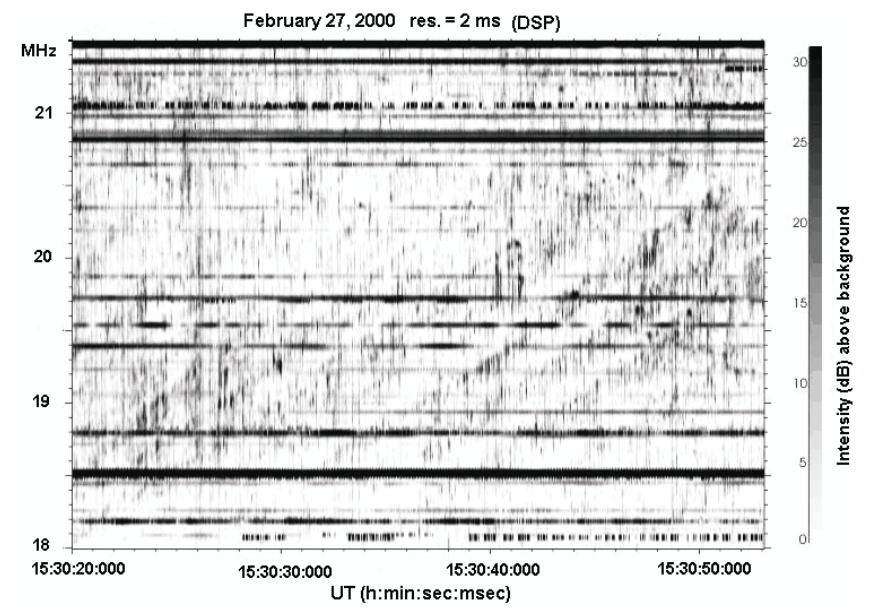

Fig. 4. Dynamic spectrum of the Jovian DAM emission with a modulation structure L2 (UTR-2 + DSP, Io-B source).

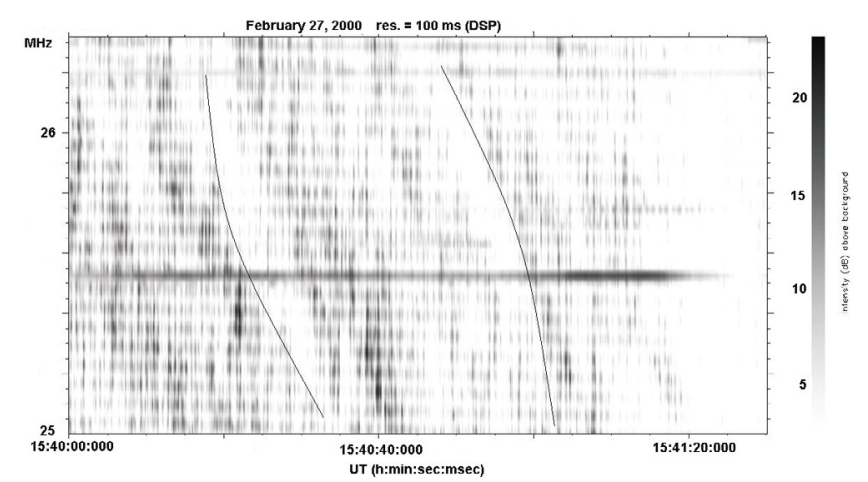

Fig. 5. Dynamic spectrum of Jovian DAM emission with L2 lanes with different signs of curvature. The different curvatures for L2 lanes are schematically marked with continuous black lines.

Jovian Central Meridian Longitude and it is the most essential argument in favor of the suggestion that this modulation effect takes place in the Jovian magnetosphere. From our observations we found the following: modulation lanes in the Io-A source $\left(\mathrm{CML} \approx 240^{\circ}\right)$ mainly have negative drift lanes L4 (80\%), and $20 \%$ of the events show a superposition of L2 and L4. The Io-B source $\left(\mathrm{CML} \approx 120^{\circ}-150^{\circ}\right)$ has $80 \% \mathrm{~L} 3$ lanes and $(5-10 \%) \mathrm{L} 2$.

A detailed analysis of much experimental data obtained with the high sensitivity receiving technique gave the following results: 1) the "modulation lanes" produced by the Jovian magnetosphere are always present in the dynamic spectra of Iodependent sources and emerge on a scale of several tens of seconds; 2) the curvature of positive (L2) and negative (L4) drifting lanes for L3 events is not always equal and even the sign of curvature can change for lanes of the same type (the radius of curvature can be simply evaluated by the magnitude of $d^{2} \mathrm{f} / d t^{2}$ ). In Fig. 3 two lines with different signs of curvature are shown with arrows. Figure 5 presents such phenomena more clearly. The different curvature for L2 lanes are schematically marked by continuous black lines.

In the more than thirty year history of "modulation lane" investigation, two main theories concerning their physical mechanism and origin have been actively discussed in the literature: a) these modulation structures occur due the Cotton-Mutton effect in Jupiter's magnetosphere and ionosphere (Gordon \& Warwick 1967; Zheleznyakov \& Shaposhnikov 1979), i.e. the result of wave propagation through a region with a transverse or 


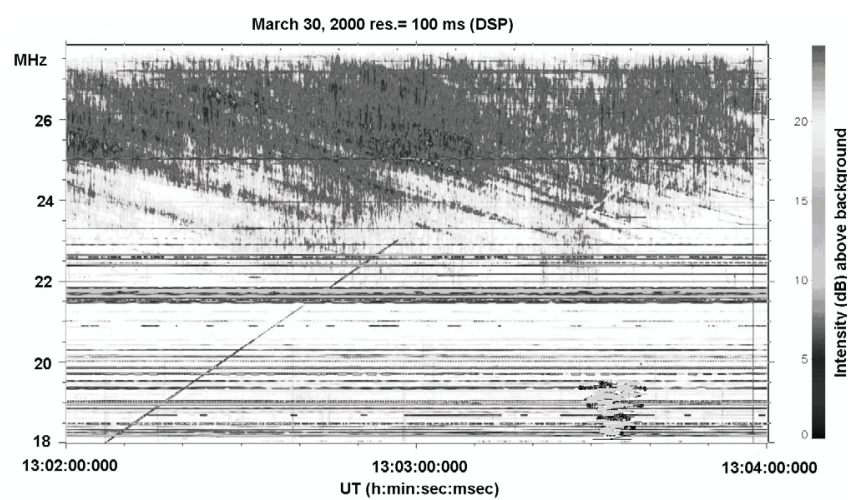

Fig. 6. Jovian DAM dynamic spectrum with "high frequency lanes" (UTR-2 + DSP, Io-B source).

quasi-transverse magnetic field. Another theory, b) assumes that modulation lanes are generated by radiation traversing a diffraction screen which is located either near the outer magnetospheric boundary (Riihimaa 1979) or in the Io plasma torus (Imai et al. 1997). At present it is still not clear if there are adequate conditions for the realization of the first hypothesis, and there is no experimental confirmation of a diffraction screen in Io's plasma torus. The Galileo plasma measurements (variation of the magnetosphere plasma behind Io at a distance $\left(\leq R_{\mathrm{Io}}\right.$, where $R_{\mathrm{Io}}$ is Io's radius) that have been reported and investigated in Russell et al. (2005) and Waldrop et al. (2005) can be associated with a diffraction structure (Arkhypov \& Rucker 2007). In Genova et al. (1981) a further theory is discussed, where the modulation lanes occur due to the wave diffraction on the drifting interplanetary disturbance. There also exists the hypothesis that the signal is modulated on the travelling disturbances in the Earth's ionosphere which can create the characteristic quasi-harmonic formations able to act as a diffraction screen for Jovian decameter radiation (Meyer-Vernet et al. 1981).

\subsection{High frequency lanes}

Genova et al. (1981) for the first time described the so-called "high frequency lanes" that cover a narrow frequency range in the highest frequency part of the spectrum. These modulations are not regular and have a shallow modulation depth. Their frequency drift is always negative. The drift rate increases with frequency. High frequency lanes often appear for the Io-B source and sometimes for the Io-A region when the CML is low. In Fig. 6 an example of the dynamic spectrum with the "high frequency lanes" obtained with UTR-2 and DSP is presented. The frequency drift of the lanes is negative with a magnitude of $0.05 \mathrm{MHz} / \mathrm{s}$ that is in accordance with that reported in Genova et al. (1981).

\subsection{Band modulation}

In the top of Fig. 7 the broad modulation bands with slow negative frequency drift $(\sim 0.02 \mathrm{MHz} / \mathrm{s})$ are presented. Zooms of some parts are given in the bottom figure. It is an example of several types of modulation "combined" in the spectra received by a terrestrial observer. In each fragment (zoom 1 and zoom 2) one can clearly see the internal structure of parallel lanes with very slow negative drifts $(\sim 0.03 \mathrm{MHz} / \mathrm{s}$ and $\sim 0.1 \mathrm{MHz} / \mathrm{s})$. Based on the hypothesis that both such structures are produced by the same diffraction screen (Imai et al. 1997), it could be possible to calculate the rate of movement of this diffraction structure

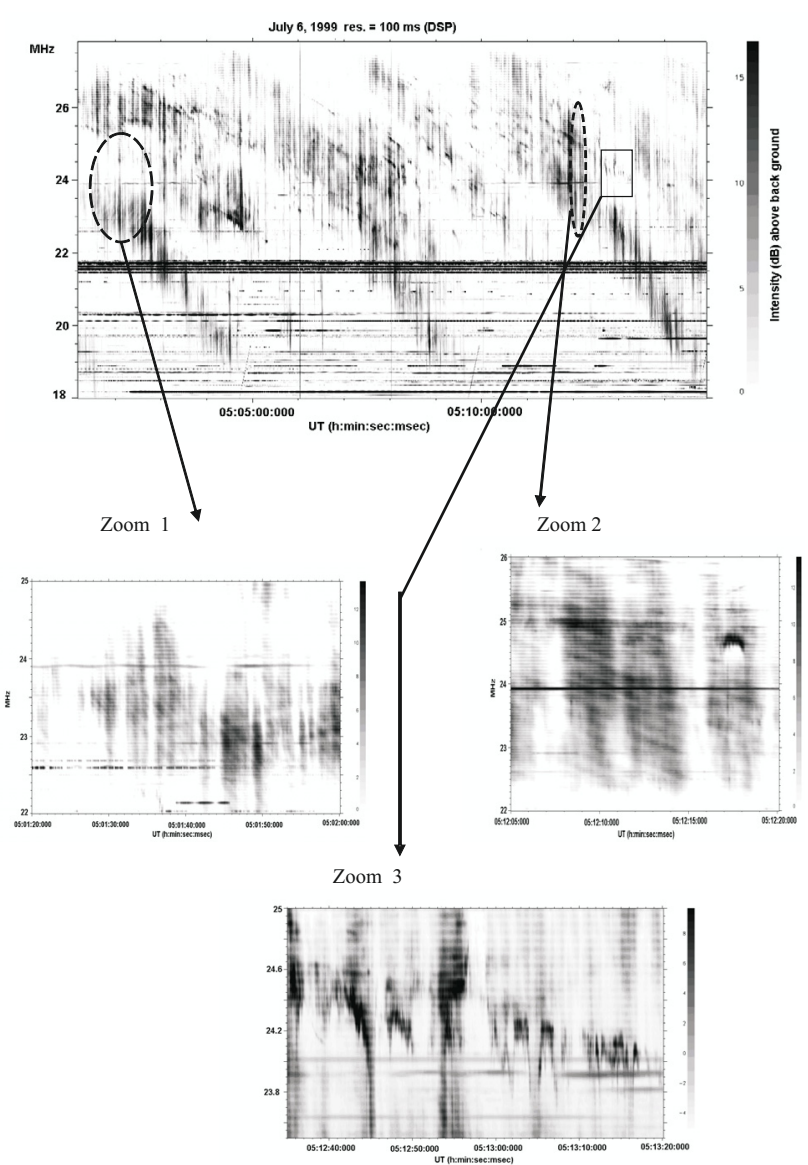

Fig. 7. Example of wide modulation lanes (top), zoom-in fragments with other kinds of modulations (bottom), (Io-C source).

and its parameter evolution. Zoom 3 of Fig. 7 once more illustrates the complexity of the Jovian DAM emission even in one short fragment of the dynamic spectrum. This fragment shows the narrow-band radiation which is analogous to an S-burst or to narrow-band emission but with an oscillating frequency band. In contrast to the earlier observed narrow-band emission with practically constant average frequency (Carr et al. 1983), in the considered case the average frequency drifts towards to the low frequencies (frequency drift is $\sim 15 \mathrm{kHz} / \mathrm{s}$ ). It is generally accepted that the Jovian decameter emission is generated at frequencies close to the local electron gyro-frequencies. Observing oscillations of the frequency band (zoom 3 of Fig. 7) show the magnetic field oscillations in the generation area (Shaposhnikov et al. 2008). With more examples like these it could be possible to investigate the internal processes in the emission source region and source parameter evolution. This is a problem for future investigations. Riihimaa supposed that the large-scale structures displayed in the top of Fig. 7 can be produced by ionospheric scintillations (Riihimaa 1988). Several kinds of modulation events (narrow and wide spectral band structures) were presented in Riihimaa (1991) and Ryabov (2001). In Riihimaa (1991) the writer considered spectral modification of the Jovian decameter S-burst storms and their internal structure in the high-resolution spectra obtained in the frequency range of $20-30 \mathrm{MHz}$ for the Io-B region. Riihimaa showed the "schedule" of S-storm development. He concluded that "there are three spectral categories that occur in different areas of the Io-B region: the L-bursts, the S-bursts and their many variants, and wide-range quasi-periodic FDS-S (Fast-Drift-Shadow-S) events", which never appear as a 


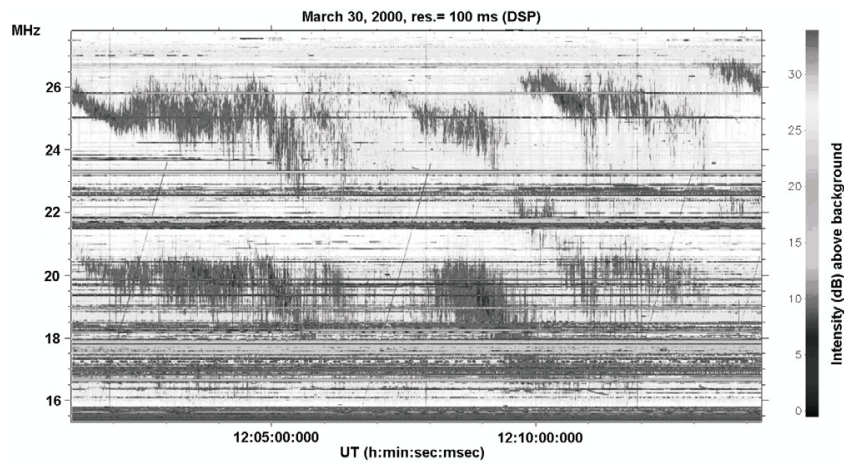

Fig. 8. Jovian DAM emission spectrum with two clearly separated spectral bands, containing a long series of S-bursts (Io-B source).

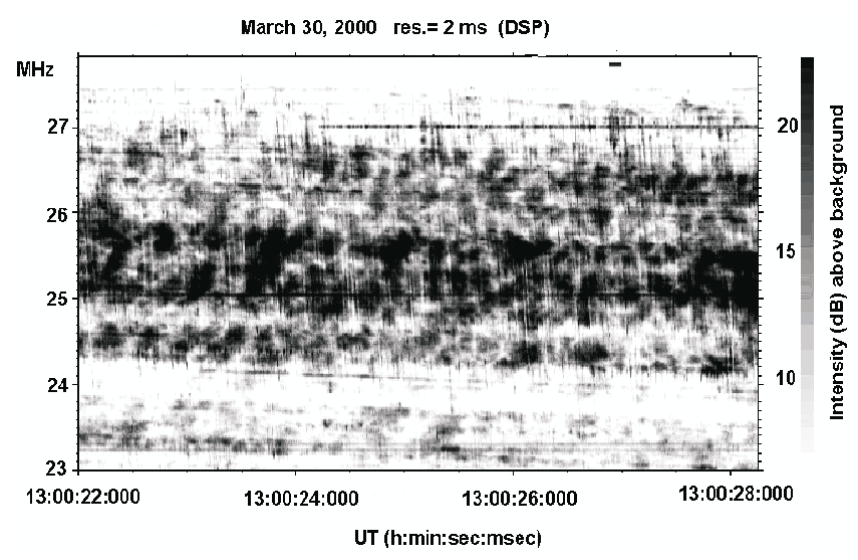

Fig. 9. An unusual dynamic spectrum: S-burst emission is modulated by intense spots with an average duration of $0.4 \mathrm{~s}$, which produced a cross-lane structure with a fast negative frequency drift of $\sim 40 \mathrm{MHz} / \mathrm{s}$ and a slow positive frequency drift of $\sim 1.2 \mathrm{MHz} / \mathrm{s}$ (Io-B source).

single burst but always as a part of a storm. Ryabov (2001) described Jupiter observations that were carried out in a frequency range of $10-30 \mathrm{MHz}$ with the UTR-2 array. He mentioned that long series of simple S-bursts are concentrated within spectral bands with a width from 1.5 to $5 \mathrm{MHz}$ and a distance between central frequencies of the bands of the order of $3.5 \mathrm{MHz}$. Bands can have a positive or a negative drift. During the experiments with UTR-2 and DSP a similar band structure was obtained (see Fig. 8). The high sensitivity and frequency-time resolution of the receiving facilities in our investigation yield more specific information and, in some cases, reveal a more complex content than was given in Riihimaa (1991) and Ryabov (2001). As an example, in Fig. 8 the dynamic spectrum with two spectral bands containing a long series of S-bursts is presented. Contrary to Riihimaa (1991) and Ryabov (2001) the distance between the central frequencies of bands is much larger and on average is $\sim 6 \mathrm{MHz}$. Also there are simultaneous variations of the whole emission frequency band. Oscillations and negative drift of emission bands is clearly seen, i.e. a strong correlation of large scale events exists. In contrast to this a correlation between small scales effects in the bands is not observed.

The spectrum presented in Fig. 9 is unique: the S-burst radiation is modulated in the form of a cross-lane structure consisting of spots of $\sim 0.4 \mathrm{~ms}$ duration. The crossed lanes demonstrate a positive and negative frequency drift simultaneously, with the faster negative drift reaching $40 \mathrm{MHz} / \mathrm{s}$ and the slow positive drift $\sim 1.2 \mathrm{MHz} / \mathrm{s}$.
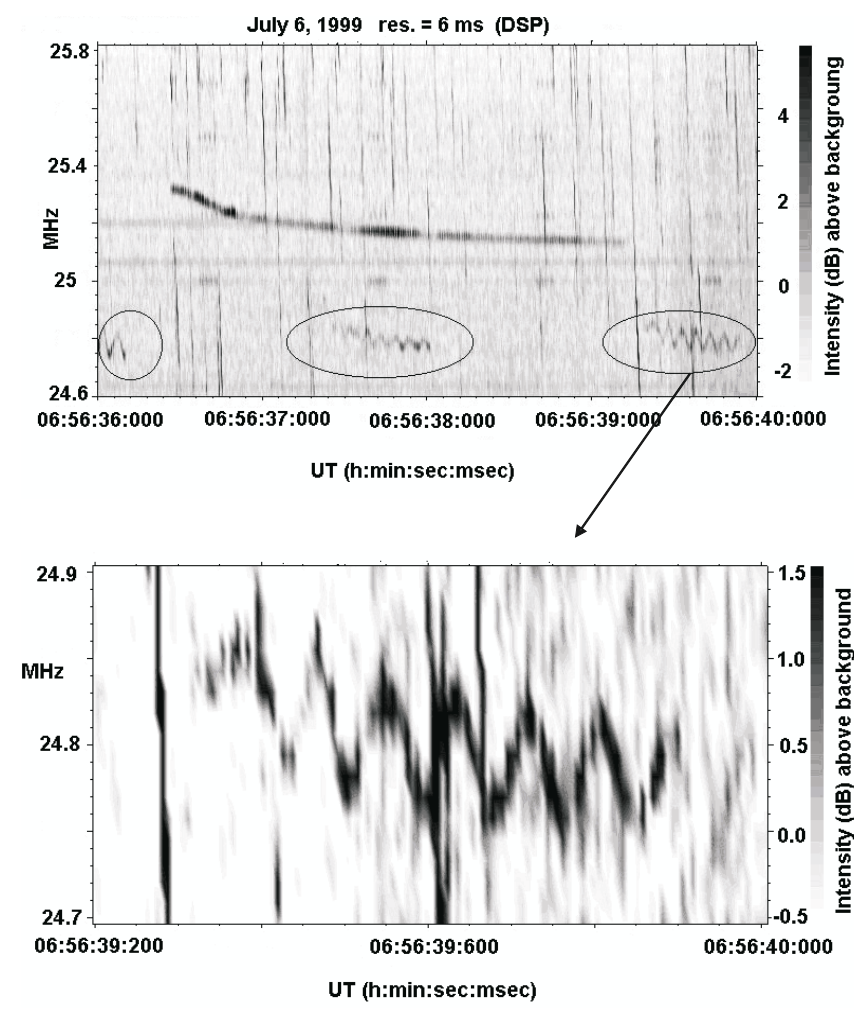

Fig. 10. Specific spectrum (Io-A source) which consists of frequency drifting consecutive quasi-harmonic structures against a background of classical S-burst emission (top), zoom-in fragment (bottom).

\subsection{Other kinds of modulation events}

In the top of Fig. 10 a more specific spectrum that consists of frequency drifting consecutive quasi-harmonic narrow-band structures against a background of classical S-burst emission is shown. The high-frequency component presents so called narrow-band events or $\mathrm{N}$ events, which have been studied in detail by Riihimaa $(1968,1977)$ as well as by other researches (see Carr et al. 1983, and references therein). The low-frequency component looks like the structure discussed above (zoom 3 of Fig. 7). It is a practically regular sinusoid, i.e. the emission shows a clear harmonic frequency modulation of the radiation band. The zoom of the low-frequency component of the event is presented in the bottom of Fig. 10. More than ten similar structures were found during the observation campaigns in 1999 and 2001. Of course, more detailed investigation needs to be carried out to determine if this phenomenon is of Jovian origin, in particular experiments in $\mathrm{ON}-\mathrm{OFF}$ regime or with distant antennae can clarify this question.

\subsection{Microstructure of a simple S-burst}

In Carr \& Reyes (1999); Litvinenko et al. (2004); Ryabov et al. (2007) the internal "fine" structure of fundamental Jovian simple S-bursts was investigated and the possible physical models for its explanation were analyzed. In Fig. 11 a characteristic example of S-burst emission data processed with the Fourier technique (top spectrum) as well as with the wavelet transform method (bottom spectrum) is shown (Litvinenko et al. 2004). A simple linear-drift $\mathrm{S}$-burst with a duration of $35 \mathrm{~ms}$ (initial data sampling rate of $50 \mathrm{MHz}$ ) was picked out from recordings of the Io-C Jovian storm (26 February 2000) obtained by the waveform receiver connected to the digital spectropolarimeter, 
installed on the UTR-2 telescope. The burst drift rate is $-27 \mathrm{MHz} / \mathrm{s}$ and its duration at a fixed frequency is approximately $1 \mu \mathrm{s}$. As can clearly be seen from the bottom panel of Fig. 11, the internal structure of the simple S-burst signal consists of very short pulses (duration $6-15 \mu \mathrm{s}$ ) which are, in turn, grouped (duration 20-150 $\mu \mathrm{s}$ ). In Carr \& Reyes (1999) these groups are called "sub-pulses". In Litvinenko et al. (2004) it was shown that "sub-pulses" have their own internal structure, i.e. micro-pulses with a duration of one separated microsecond pulse: $6-15 \mu \mathrm{s}$, instantaneous frequency band of one separated microsecond pulse $100-300 \mathrm{kHz}$ and time interval between consecutive microsecond pulses 5-25 $\mu \mathrm{s}$. Moreover it was found that each pulse occupies a specific frequency interval, which we mentioned above as the instantaneous frequency band, but practically has no clearly defined frequency drift with time. This shows that the frequency drift of a simple burst as a whole results from frequencies decreasing with time for each subsequent pulse. According to our present knowledge about the scales of the processes in the Jovian magnetosphere, the interplanetary medium, the Earth magnetosphere and ionosphere, it can be affirmed that these microsecond structures are not modulated along the propagation path from the source region to the observer. In Carr \& Reyes (1999) two interesting possible empirical models of the obtained S-burst microstructure have been proposed: 1) the "narrow-band fluctuation model" that assumes that the burst waveform represents the statistical fluctuations of the sum of the instantaneous amplitudes of a large number of closely spaced but randomly phased frequency components; 2) the "pulse superposition model" which is based on the assumption that the S-burst signal represents the superposition of a group of pulses from closely spaced but independent short-lived emission centers which are nearly monochromatic but slightly different in frequency. It is now generally accepted that the Jovian decametric radiation is represented by fast extraordinary waves and its emission mechanism is coherent. In this context the S-burst radiation is usually connected to the instabilities of electromagnetic and plasma waves near the upperhybrid frequency which is caused by groups of hot electrons with different anisotropic distribution functions. When the generation mechanism of an extraordinary wave is studied, two possible processes should be considered: 1) the direct excitation of such a wave by non-equilibrium particles in the source (cyclotronmaser mechanism); 2) the generation of a plasma wave near the upper-hybrid frequency with a subsequent transformation into a fast extraordinary wave (plasma mechanism). On the basis of these ideas a number of possible generation models were considered in Litvinenko et al. (2004, and references therein).

In Ryabov et al. (2007) a further study of sub-pulses appearing as $\sim 4-150 \mu$ s timescale fluctuations of the narrow-band oscillations envelope was discussed. The concept of a narrowband random Gaussian noise process with a slowly changing envelope and phase was suggested as a mathematical model of S-burst radiation capable of reproducing the recorded properties of emission. The writers concluded that for this phenomenon the "narrow-band amplifier" model gives the best fit to the observed parameters. It was also underlined that this process does not require any additional special mechanism (for instance, oscillations or superimposed modulations). All the proposed models (Carr \& Reyes 1999; Litvinenko et al. 2004; Ryabov et al. 2007) need further detailed investigation of statistical and physical characteristics long series of waveform high-sensitivity observations (unfortunately, in Litvinenko et al. 2004; Ryabov et al. 2007, relatively short waveform realizations were considered, $6 \mathrm{~s}$ and $900 \mathrm{~s}$, correspondingly).
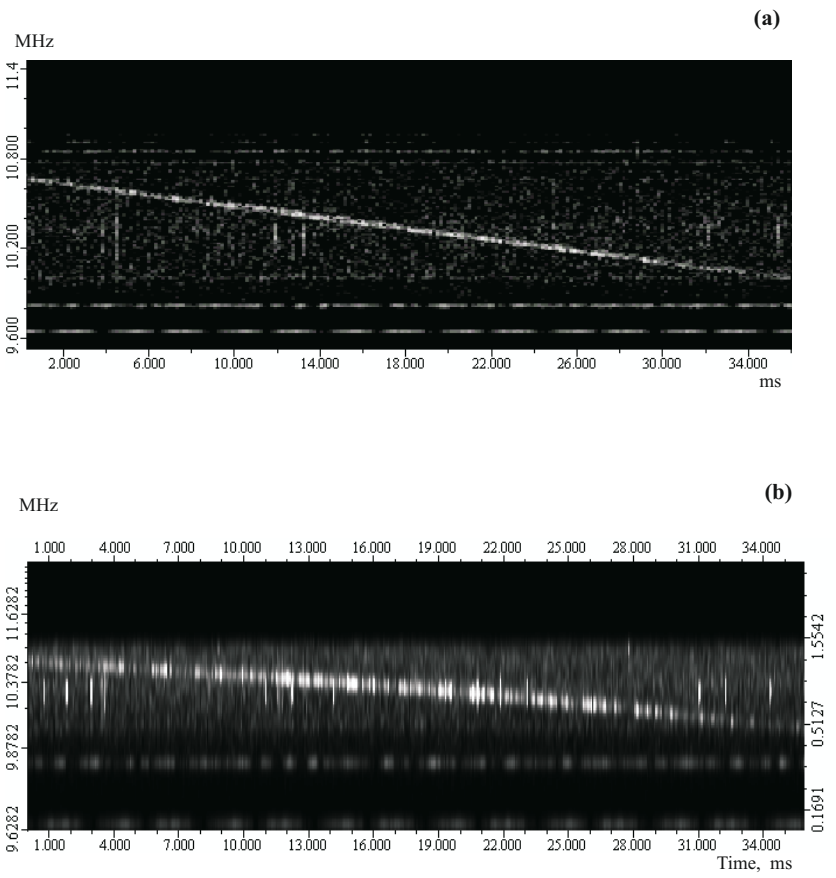

Fig. 11. a) Fourier spectrum of a single S-burst (Io-C source), b) wavelet spectrum of the same burst.

One more example of the complex internal structure of S-bursts (Io-B source) is shown in Fig. 12. The time-frequency resolution in the top panel (a) is $8 \mathrm{kHz}$ and $128 \mu$ s that allows us to determine the bursts in a Z-envelope form. In the bottom panel a fragment of the same spectrum with a time resolution of $4 \mu \mathrm{s}$ and a frequency resolution $250 \mathrm{kHz}$ demonstrates the superfine structure of the S-burst. An analysis of the spectrum envelope shows that the emission starts at point $\mathrm{z}$ and then develops as narrow-band radiation with $\Delta f_{1} \simeq 10 \mathrm{kHz}$ and negative frequency drift as well as radiation with $\Delta f_{2} \simeq 100 \mathrm{kHz}$ and zero frequency drift. It is supposed that S-bursts with negative frequency drift can be attributed to the cyclotron-maser emission of electrons in the magnetic flux tubes that connect Io and Jupiter. Generation of radiation takes place at the local gyrofrequency of electrons moving from Jupiter to Io according to electron adiabatic motion in the magnetic field. In our case a horizontal branch of the Z-envelope indicates that for $10 \mathrm{~ms}$ a part of the electron flow is in the magnetic instability zone with a quasistable field. This instability can be caused by azimuth electric currents in the Jovian magnetosphere at heights of $1.1-1.2 R_{\mathrm{J}}$ which distort the magnetic field of the planet.

\subsection{Simultaneous observations}

A comparison of S-burst radiation recorded simultaneously at two different observatories would allow a study of the special emission geometry of Jupiter's decametric radio component. The idea is to search for spectral differences on the basis of the correlation analysis in simultaneously recorded S-burst patterns which may result from an emitting hollow cone structure sweeping over both observatories.

Figure 13 displays the S-emission recorded simultaneously with the DSP receiver in Nancay and in Kharkov, over a period of $6 \mathrm{~s}$. As can be seen, the given time resolution $(6 \mathrm{~ms}$ in Kharkov, $2 \mathrm{~ms}$ in Nancay) and frequency resolution $(12.2 \mathrm{kHz})$ are far too coarse to eliminate the influence of the Faraday effect that splits a linear S-burst into "bubbles" of increasing and 


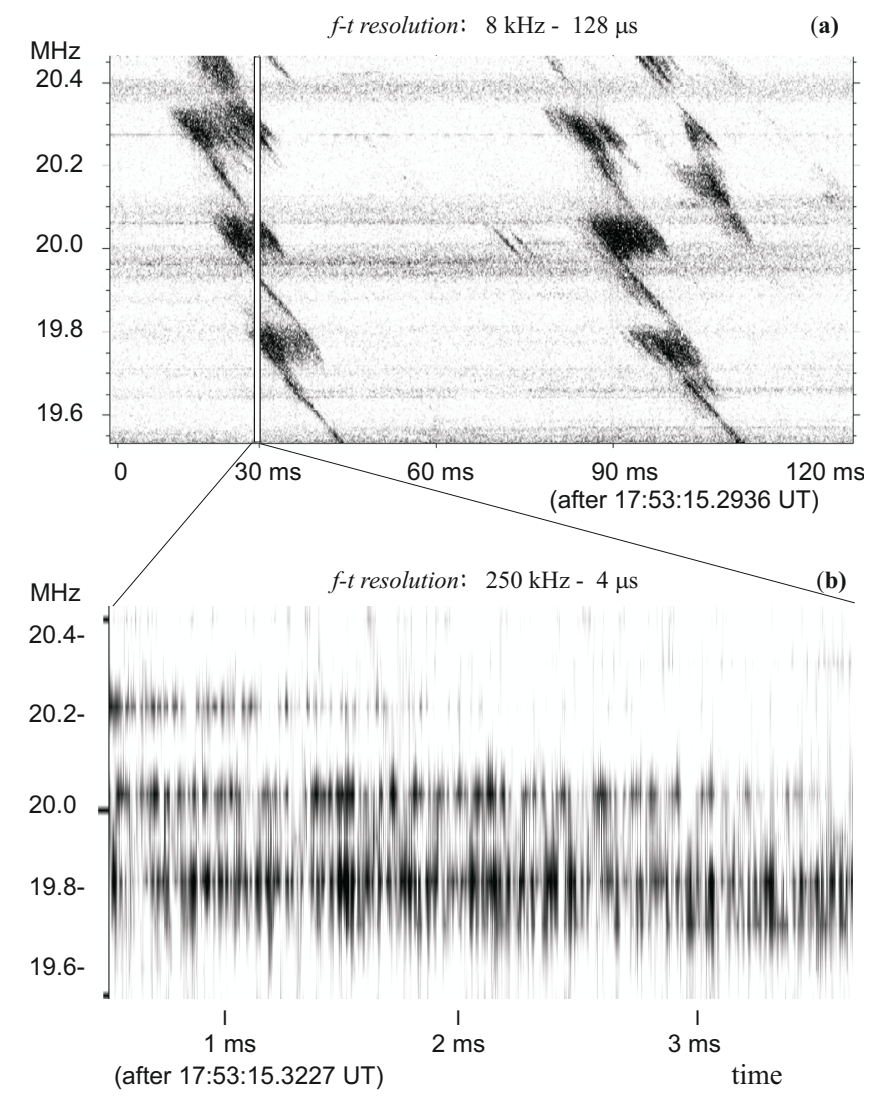

Fig. 12. a) Z-envelope dynamic spectra (UTR-2 +DSP, Io-B source), b) "superfine" structure of the S-burst.

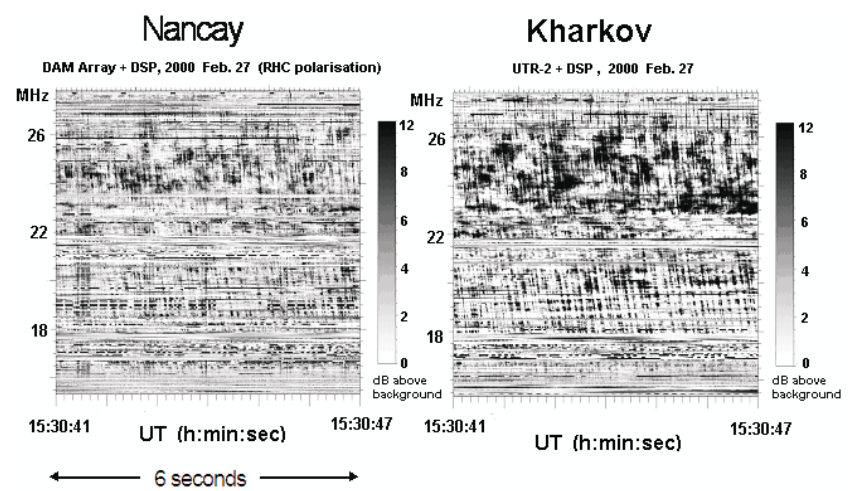

Fig. 13. Synchronized, distant observations of the Jupiter DAM, UTR-2 - NDA, $3000 \mathrm{~km}$ longitudinal separation, (Io-B source).

decreasing intensities. A much higher spectral resolution, for instance as provided by the waveform receiver, will be required to bypass the influence of the Earth's ionosphere and to enter the domain of S-burst "sub-pulses".

It will be interesting to analyse if successive sub-pulses within a narrow-band frequency range share the same cone structure with stable parameters over time or if the emission geometry changes from one sub-pulse to the next. In this case, high resolution sub-pulse spectra will look slightly different from Kharkov and from Nancay because a sub-pulse's hollow cone structure will not always arrive at both stations simultaneously.

The technical requirements for this kind of analysis are high and will require new large antenna arrays like the future LOFAR project.

\section{Conclusion}

In the present work the wide-band dynamic spectra of the Jovian decameter emission obtained over the last decade with highfrequency and high-time resolution equipment on the UTR-2 radio telescope have been analyzed. Attention was paid to detecting different kinds of modulation effects: those already known and also newly discovered. The high sensitivity, high-time and high-frequency resolution of the equipment reveal the hierarchy of modulation, "size scaled" in the time and frequency domains. As a result, a set of "fine" structures appears on the Jovian dynamic spectra at time scales shorter $\sim 10 \mathrm{~min}$. It was concluded that practically all observed radiation is in some way modulated. In the present work we obtained several new results. For instance, it was emphasized that the "modulation lanes" produced by the Jovian magnetosphere always appear on the scale of minutes, while the data time resolution is about $100 \mathrm{~ms}$. The distribution of positive and negative frequency drift lanes for Io-A and Io-B sources was evaluated. It was shown that the curvature of positive and negative drifting lanes for one L3 event is not always equal, and the sign of curvature can change for the same lanes. New intriguing spectrum modulation has been found on the scale of seconds, such as S-burst radiation which is modulated in the form of a cross-lane structure consisting of spots, the negative frequency drifting consecutive quasi-harmonic structure existing against a background of classical S-burst emission, the narrow-band emission with an oscillating frequency band, etc. The properties of the linear drifting simple S-burst microstructure also were discussed.

The telescopes and receiving equipment used in the experiments have optimal characteristics for a spectral investigation of the Jovian decameter emission. Further progress may be made using the new giant LOw Frequency antenna ARray (LOFAR) and Low Wavelength Array (LWA) (de Pater et al. 2004; van Haarlem 2005). The Jovian programme is of interest to the LOFAR creators (see, for instance, Zarka 2004; van Haarlem 2005; Nigl et al. 2007, and LOFAR scientific Application, Report ASTRON-LOFAR-00230). Moreover, in January 2008 the creation of very large low frequency antennae $(10-80 \mathrm{MHz})$ was debated at the LOFAR Super Station Meeting in Paris, France. Such antennae are planned to be developed in France (www . lesia. obspm. fr/plasma/LOFAR) and in Ukraine (Konovalenko et al. 2005).

Acknowledgements. The authors thank Prof. Yu. Jampolsky, Dr. V. Galushko and Dr. V. Sinitsin for fruitful discussions and help during the preparation of this article. The work was supported by the Commission on International Collaboration of the Austrian Academy of Sciences and also by the Russian Foundation for Basic Research (grant 08-02-00119-a).

\section{References}

Arkhypov, O. V., \& Rucker, H. O. 2007, A\&A, 467, 353

Boischot, A., Rosolen, C., Aubier, M., et al. 1981, Icarus, 43, 399

Boudjada, M. Y., \& Lecacheux, A. 1991, A\&A, 247, 235

Braude, S. I., Men, A. V., \& Sodin, L. G. 1978, Antenny, 26, 3

Burke, B. F., \& Franklin, K. L. 1955, J. Geophys. Res., 60, 213

Calvert, W., Leblanc, Y., \& Ellis, G. R. A. 1988, ApJ, 335, 976

Carr, T. D., \& Reyes, F. 1999, J. Geophys. Res., 104, 25127

Carr, T. D., Desh, M. D., \& Alexander, J. K. 1983, in Physics of the Jovian magnetosphere, ed. A. J. Dessler (Cambridge University Press), 226

Daubechies, I. 1988, Commun. Pure Appl. Math., 41, 909

de Pater, I., Kassim, N., \& Rucker, H. 2004, Planet. Space Sci., 52, 1339

Flagg, R. S., \& Desch, M. D. 1979, J. Geophys. Res., 84, 4238

Genova, F., \& Leblanc, Y. 1981, A\&A, 98, 133

Genova, F., Aubier, M. G., \& Lecacheux, A. 1981, A\&A, 104, 229

Goertz, C. K. 1976, J. Geophys. Res., 81, 3368

Gordon, M. A., \& Warwick, J. M. 1967, ApJ, 148, 511 
Herbert, F., Sandel, B. R. \& Broadfoot, A. L. 1967, J. Geophys. Res., 72, 4773 Hess, S., Mottez, F., \& Zarka, P. 2007a, J. Geophys. Res., 112, A11212

Hess, S., Zarka, P., \& Mottez, F. 2007b, Planet. Space Sci., 55, 89

Hospodarsky, G. B., Kurth, W. S., Cecconi, B., et al. 2004, J. Geophys. Res., 109, A09S07

Imai, K, Wang, L., \& Carr, T. D. 1997, J. Geophys. Res, 102, 7127

Kleewein, P., Rosolen, C., \& Lecacheux, A. 1997, in Planetary Radio Emissions IV: Proceedings of the 4th international workshop, Graz, Austria, September 9-11, 1996, ed. H. O. Rucker, S. J. Bauer, \& A. Lecacheux (Austrian Academy of Sciences Press), 349

Konovalenko, A. A., Lecacheux, A., Rosolen, C., \& Rucker, H. O. 2001, in Planetary Radio Emissions V: Proceedings of the 5th international workshop, Graz, Austria, April 2-4, 2001, ed. H. O. Rucker, M. L. Kaiser, \& Y. Leblanc (Austrian Academy of Sciences Press), 63

Konovalenko, A. A., Falkovich, I., Kalinichenko, N., et al. 2005, Exp. Astron., 16,149

Ladreiter, H. P., Litvinenko, G. V., Boudjada, M. Y., \& Rucker, H. O. 1995, Planet. Space Sci., 43, 1595

Lecacheux, A. 1976, A\&A, 49, 197

Leitner, M., \& Rucker, H. O. 2001, in Planetary Radio Emissions V: Proceedings of the 5th international workshop, Graz, Austria, April 2-4, 2001, ed. H. O. Rucker, M. L. Kaiser, \& Y. Leblanc (Austrian Academy of Sciences Press), 91

Litvinenko, G. V., Rucker, H. O., Vinogradov, V. V., Rybov, B. P., \& Shaposhnikov, V. E. 2000, Radio Sci., 35, 1445

Litvinenko, G. V., Rucker, H. O., Vinogradov, V. V., Leiner, M., \& Shaposhnikov, V. E. 2004, A\&A, 426, 343

Melrose, D. B. 1986, J. Geophys. Res., 91, 7970

Meyer-Vernet, N., Daigne, G., \& Lecacheux, A. 1981, A\&A, 96, 296

Mutel, R. L., Christopher, I. W., \& Peterson, W. W. 2007, in American Geophysical Union, Fall Meeting 2007 (American Geophysical Union), SM53A-1091

Nigl, A., Zarka, P., Kuijpers, J., et al. 2007, A\&A, 471, 1099

Parker, G. D., Dulk, G. A., \& Warwick, J. W. 1969, ApJ, 157, 439

Riihimaa, J. J., Dulk, G. A., \& Warwick, J. W. 1970, ApJS, 19, 175

Riihimaa, J. J. 1968, AJ, 73, 265

Riihimaa, J. J. 1970, A\&A, 4, 180

Riihimaa, J. J. 1977, Ap\&SS, 51, 363

Riihimaa, J. J. 1978, Ap\&SS, 56, 503

Riihimaa, J. J. 1979, A\&A, 78, L21

Riihimaa, J. J. 1988, Earth, Moon, and Planets, 42, 303

Riihimaa, J. J. 1991, Earth, Moon, and Planets, 53, 157
Rucker, H. O., Lecacheux, A., Konovalenko, A. A., \& Leitner, M. V. 2001, in Planetary radio emission V: Proceedings of the 5th international workshop, April 2-4, 2001, Graz, Austria, ed. H. O. Rucker, M. L. Kaiser, \& Y. Leblanc (Graz: Austrian Academy of Sciences Press), 51

Russell, C. T., Kivelson, M. G., \& Khurana, K. K. 2005, Planet. Space Sci., 53, 937

Ryabov, B. P. 1992, in Planetary Radio Emission III: Proceedings of the 3th international workshop, September 2-4, 1991, Graz, Austria, ed. H. O. Rucker, S. J. Bauer, \& M. L. Kaiser (Vienna: Austrian Academy of Sciences Press), 125

Ryabov, B. P. 2001, Radiophys. Radioastron., 6, 103

Ryabov, B. P., \& Gerasimova, N. N. 1990, Sporadic radio emission of Jupiter at decameter wavelength (Kiev: Naukova Dumka)

Ryabov, V. B., Ryabov, B. P., Vavriv, D. M., et al. 2007, J. Geophys. Res., 112, A09206

Shaposhnikov, V. E., Boudjada, M. Y., Rucker, H. O., Zaitsev, V. V., \& Aubier, M. 1999, A\&A, 344, 709

Shaposhnikov, V., Kostrov, A., Guschin, M., et al. 2008, in 30th General Assembly EGU: Geophysical Research Abstracts, Vol. 2 (European Geophysical Society), 141

Spilker, T. R., Senske, D. A., Prockter, L., et al. 2007, in American Geophysical Union, Fall Meeting 2007 (American Geophysical Union), P21B-0532

Taubenschuss, U., Rucker, H. O., \& Macher, W. 2006, in Planetary radio emission VI: Proceedings of the 6th international workshop, Graz, Austria, April 20-22, 2005, ed. H. O. Rucker, W. S. Kurth, \& G. Mann (Austrian Academy of Sciences Press), 175

van Haarlem, M. P. 2005, EAS Publ. Ser., 15, 431

Waldrop, L. S., Fritz, T. A., Kivelson, M. G., et al. 2005, Planet. Space. Sci., 53, 681

Warwick, J. W., \& Dulk, G. A. 1964, Science, 145, 380

Willes, A. J. 2002, J. Geophys. Res., 107, SMP 7

Zaitsev, V. V., Zlotnik, E. Y., \& Shaposhnikov, V. E. 1986, A\&A, 169, 345

Zarka, P. 1998, J. Geophys. Res., 103, 20159

Zarka, P. 2004, Adv. Sp. Res., 33, 2045

Zarka, P. 2006, in Planetary radio emission VI: Proceedings of the 6th international workshop, Graz, Austria, April 20-22, 2005, ed. H. O. Rucker, W. S. Kurth, \& G. Mann (Austrian Academy of Sciences Press), 543

Zarka, P., Hess, S., Ray, L. C., Cecconi, B., \& Mottez, F. 2007, in American Geophysical Union, Fall Meeting 2007 (American Geophysical Union), SM53A-1087

Zheleznyakov, V. V., \& Shaposhnikov, V. E. 1979, SvA, 23, 588 